\title{
Improved Gaussian Mixture Probability Hypothesis Density for Tracking Closely Spaced Targets
}

\author{
Huanqing Zhang, Hongwei Ge, and Jinlong Yang
}

\begin{abstract}
Probability hypothesis density (PHD) filter is a suboptimal Bayesian multi-target filter based on random finite set. The Gaussian mixture PHD filter is an analytic solution to the PHD filter for linear Gaussian multi-target models. However, when targets move near each other, the GM-PHD filter cannot correctly estimate the number of targets and their states. To solve the problem, a novel reweighting scheme for closely spaced targets is proposed under the framework of the GM-PHD filter, which can be able to correctly redistribute the weights of closely spaced targets, and effectively improve the multiple target state estimation precision. Simulation results demonstrate that the proposed algorithm can accurately estimate the number of targets and their states, and effectively improve the performance of multi-target tracking algorithm.
\end{abstract}

Keywords - closely spaced targets, random finite set, probability hypothesis density filter, Gaussian mixture PHD, weight redistribution

\section{INTRODUCTION}

$\mathbf{I}$ $\mathrm{N}$ recent years, the random finite set (RFS) theory [1] for tracking multiple targets has attracted considerable attention, which offers an elegant representation of a finite, time-vary number of targets and measurements. Both the probability hypothesis density (PHD) [2] and the cardinality PHD (CPHD) [3] are suboptimal approximation but more tractable alternative to the RFSs Bayesian multiple target filtering. Two major implementations of the PHD filter are particle PHD [4] and Gaussian mixture PHD [5]. Moreover, there are some modified versions of both PHD and CPHD in [6-8].

For the problem of closely spaced target tracking, there are some approaches reported in the literature. In [9], a dynamic detection probability method is introduced into the GM-PHD filter, which is used to compute the detection probability of individual targets. However, the size of each target must be known as prior, and the size of all targets must be same. The two factors make the algorithm not to be widely applicable. In [10-12], the labeled RFSs is introduced into the multi-Bernoulli filter to address target trajectories and their uniqueness, and can

This work was supported by the National Natural Science Foundation of China (No. 61305017), the Natural Science Foundation of Jiangsu Province (No. BK20130154), and the key scientific research project of higher education institution of Henan province(No. 16A520066).

H.Q. Zhang is with School of Electronic \& Electrical Engineering, Shangqiu Normal University, China and with School of Internet of Things Engineering, Jiangnan University, China (e-mail: hsj_hqzhang@126.com).

H.W. Ge is with School of Internet of Things Engineering, Jiangnan University, China (e-mail: rainfall99@126.com). *: Corresponding author.

J.L. Yang is with School of Internet of Things Engineering, Jiangnan University, China (e-mail: jinlyang@yeah.net). achieve better performance when targets move near each other. Unfortunately, the labeled RFSs filters required super-exponential growth of the number of components to adequately represent the multi-target states, and more complexity than the PHD filter. In [13, 14], a PGM-PHD filter is proposed to solve the problem of tracking closely spaced targets, where a penalized weight competition method is adopted under the framework of the GM-PHD filter. The penalized method is used to refine the weights of closely spaced targets in the update step of the GM-PHD filter, and the PGM-PHD filter gets over the defect of the GM-PHD filter. Unfortunately, the PGM-PHD filter is not able to recognize the identity of individual target, and the track estimation of individual target cannot be obtained directly. In order to solve this problem, a collaborative penalized Gaussian mixture PHD (CPGM-PHD) filter is proposed in [15], which utilizes different identity of individual target to collaboratively penalize the weights of closely spaced targets. The CPGM-PHD filter not only improves the estimation accuracy of both the target number and their states, but also provides target trajectories over time. However, the penalized weight scheme of both the PGM-PHD and CPGM-PHD filter has the defect that the weights of closely spaced targets cannot be solved completely.

To solve the problem of tracking closely spaced targets, an improved Gaussian mixture probability hypothesis density algorithm is proposed, where a novel target reweighting method is utilized to alleviate the weight error of closely spaced targets.

The remainder of this paper is organized as follows. Section 2 explains the background of multi-target tracking and illustrates the problem of closely spaced targets. The proposed multi-target tracking algorithm is discussed in Section 3. In Section 4, we study the performance of the proposed approach via different Monte Carlo simulations. Finally, the conclusions are given in Section 5.

\section{PROBLEM ForMULATION}

\section{-A. PHD Filter}

In RFS theoretical framework, the multi-target states and multi-target observations defined as random finite sets are $X_{k}=\left\{x_{k, 1}, x_{k, 2}, \ldots, x_{k, N_{k}}\right\}$ and $Z_{k}=\left\{z_{k, 1}, z_{k, 2}, \ldots, z_{k, M_{k}}\right\}$, where the $N_{k}$ and $M_{k}$ denote the target number and measurement number at time $k$, respectively.

The PHD filter is a suboptimal alternative to multi-target Bayesian filter, which propagates the first order statistical moment of the posterior multi-target states. The PHD filter 
recursive calculation consists of prediction step and update step. The prediction equation is

$$
\begin{aligned}
\mathcal{V}_{k \mid k-1}(x)= & \int p_{s, k}(\zeta) f_{k \mid k-1}(x \mid \zeta) \mathcal{V}_{k-1}(\zeta) d \zeta \\
& +\int \beta_{k \mid k-1}(x \mid \zeta) \mathcal{V}_{k-1}(\zeta) d \zeta+\gamma_{k}(x)
\end{aligned}
$$

When the latest measurement set $Z_{k}$ is available at time $k$, the PHD update equation can be described as

$$
\begin{aligned}
\mathcal{V}_{k}(x)= & {\left[1-p_{D, k}(x)\right] \mathcal{V}_{k \mid k-1}(x)+} \\
& \sum_{z \in Z_{k}} \frac{p_{D, k}(x) g_{k}(z \mid x) \mathcal{V}_{k \mid k-1}(x)}{\kappa(z)+\int p_{D, k}(\zeta) g_{k}(z \mid \zeta) \mathcal{V}_{k \mid k-1}(\zeta) d \zeta}
\end{aligned}
$$

where $p_{S, k}$ is the survival probability, $p_{S, k}$ is the detection probability, and $\kappa(z)$ is the clutter intensity. $\gamma_{k}(x)$ is the intensity function of the newborn targets, and $\beta_{k \mid k-1}(\square \zeta \zeta)$ is the spawn target intensity.

\section{B. Gaussian Mixture PHD Filter}

Although the PHD filter can greatly reduce the calculation load of multiple targets Bayesian filter, it is still need to be approximated by some numerical method. Gaussian mixture PHD (GM-PHD) filter can provide a closed-form solution through the summation of mixing weights of Gaussian components to approximate the PHD function.

Let $\quad(; m, P)$ illustrate a Gaussian density with mean $m$ and covariance $P$. Assume the posterior intensity at time $k$ is expressed as a Gaussian mixture with $J_{k-1}$ components as

$$
v_{k-1}(x)=\sum_{i=1}^{J_{k-1}} w_{k-1}^{(i)} \quad\left(x ; m_{k-1}^{(i)}, P_{k-1}^{(i)}\right)
$$

where $w_{k-1}^{(i)}$ is the weight of ith Gaussian mixture at time $k-1$. Then, the predicted intensity at time $k$ is also a Gaussian mixture with $J_{k \mid k-1}$ components calculated as

$$
v_{k \mid k-1}(x)=\sum_{i=1}^{J_{k \mid k-1}} w_{k \mid k-1}^{(i)} \quad\left(x ; m_{k \mid k-1}^{(i)}, P_{k \mid k-1}^{(i)}\right)
$$

When the measurement set $Z_{k}$ is available at time $k$, the posterior intensity at time $k$ is a Gaussian mixture and can be described as

$$
v_{k}(x)=\left(1-p_{D, k}\right) v_{k \mid k-1}(x)+\sum_{z \in Z_{k}} \sum_{i=1}^{J_{k \mid k-1}} w_{k}^{(i)}(z) \quad\left(x ; m_{k \mid k}^{(i)}(z), P_{k \mid k}^{(i)}\right)
$$

where $w_{k}^{(i)}$ denotes the weight of ith target computed as

$$
w_{k}^{(i)}(z)=\frac{p_{D, k} w_{k \mid k-1}^{(i)} g\left(z \mid x^{(i)}\right)}{\kappa(z)+p_{D, k} \sum_{j=1}^{J_{k k-1}} w_{k \mid k-1}^{(j)} g\left(z \mid x^{(j)}\right)}
$$

The posterior PHD is propagated by the PHD recursion similar to Kalman filter. Detail process of the GM-PHD filter can be referred to [5].

\section{The Defects of the GM-PHD Filter}

The performance of the GM-PHD filter degrades dramatically when targets are in close proximity, such as crossing or paralleling targets. The GM-PHD filter utilizes a weighted summation of Gaussian components to estimate the targets state in time, and extracts the state of targets based on a given state extraction threshold. At each iterative update step, the estimation of individual target may be lost when the weight of the individual target below the given state extraction threshold. In closely spaced targets tracking scenario, the major reason of this phenomenon is that the corresponding measurements of these targets are not available at the update step. Take, for instance, two targets into account. The problem is graphically illustrated in Fig.1.

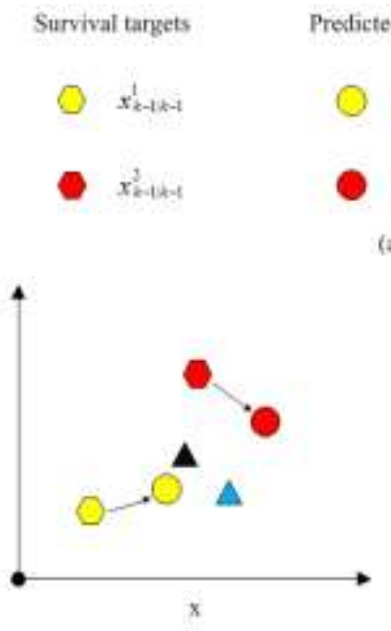

(b)

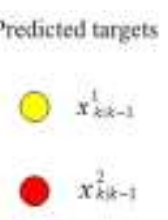

(a)

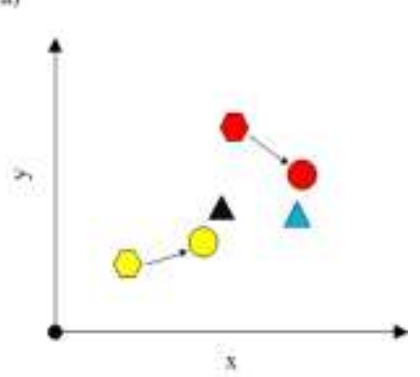

(c)
Fig. 1. Position relationship between predicted targets and measurements. (a) Symbol representation for targets and measurements. (b) position relationship between predicted targets and measurements. (c) one of possible and reasonable position relationship between predicted targets and measurements

Suppose that two targets with the state $x_{k-1 \mid k-1}^{1}$ and $x_{k-1 \mid k-1}^{2}$ survive at time $k-1$. Two predicted targets with the state $x_{k \mid k-1}^{1}$ and $x_{k \mid k-1}^{2}$ stem from survival targets $x_{k-1 \mid k-1}^{1}$ and $x_{k-1 \mid k-1}^{2}$, respectively, meanwhile two measurements $z_{k}^{a}$ and $z_{k}^{b}$ are generated at time $k$. In order to analyse conveniently, suppose that the clutter rate is zero, and the detection probability is one. In the update step of the GM-PHD filter, each predicted target is updated with the two measurements. So, four targets are created including $x_{k}^{1, a}, x_{k}^{1, b}, x_{k}^{2, a}$ and $x_{k}^{2, b}$, where $x_{k}^{m, n}$ shows the state of a target generated by updating the mth target with the $n t h$ measurement.

Let $w_{k}^{m, n}$ be the weight of the target $x_{k}^{m, n}$ after the update step of GM-PHD, where $m$ and $n$ are the same above. As is shown in Fig.1(b), because both the two measurements are closer to the predicted target $x_{k \mid k-1}^{1}$ comparing to the predicted target $x_{k \mid k-1}^{2}$, therefore $w_{k}^{1, a}$ and $w_{k}^{1, b}$ are much greater than $w_{k}^{2, a}$ and $w_{k}^{2, b}$, and similarly the corresponding normalized weight $\bar{w}_{k}^{1, a}$ and $\bar{w}_{k}^{1, b}$ are much greater than $\bar{w}_{k}^{2, a}$ and $\bar{w}_{k}^{2, b}$. For example, suppose that the normalized weights $\bar{w}_{k}^{1, a}=0.8$, $\bar{w}_{k}^{1, b}=0.65, \quad \bar{w}_{k}^{2, a}=0.2$, and $\bar{w}_{k}^{2, b}=0.35$, respectively. As a result, the two targets $x_{k}^{1, a}$ and $x_{k}^{1, b}$ are extracted as the estimation results in that the weights of the two targets above a given extraction threshold $w_{T h}$ (generally $w_{T h}>0.5$ ).

In multiple target tracking, it is assumed that one target only generate one measurement and vice visa, named one-to-one rule. The one-to-one rule means that a target can only use one measurement in the update step. If a target confirms the rule, 
the target can have only one effective state where the corresponding normalized weight above the threshold $w_{T h}$, otherwise, the target violates the one-to-one rule. As the example illustrated above, the GM-PHD filter break out one-to-one rule when targets move near each other. The two targets $x_{k}^{1, a}$ and $x_{k}^{1, b}$ evolve from the target $x_{k \mid k-1}^{1}$ at time $k$. Under the one-to-one rule, the reasonable estimation result should be composed of the targets evolving from different predicted targets. That is, one evolves from the target $x_{k \mid k-1}^{1}$, and other is originated from the target $x_{k \mid k-1}^{2}$.

\section{The Proposed Multi-TARget Algorithm}

As is illustrated in Section 2.3, the GM-PHD filter cannot correctly update each target when targets move near each other. One of possible and reasonable position relationship between predicted targets and measurements is demonstrated in Fig.1(c). If there is a strategy that can update closely spaced targets by using the measurements shown in Fig.1(b) to achieve the same result as using the measurements in Fig.1(c) in update step of the GM-PHD filter, then the problem of closely spaced targets can be solved. In this section, a novel reweighting scheme is incorporated into the GM-PHD filter to redistribute the incorrect weights of closely spaced targets.

Assuming that the predicted multi-target intensity can be approximated by Eq.(4), and the multi-target measurement set $Z_{k}=\left\{z_{n, k}\right\}_{n=1}^{N_{k}}$ is available at time $k$. For the purpose of distinguishing individual target, an unique label is assigned for each target, which is denoted by $\ell$. Each Gaussian components of the individual target has the same label. In the update step of the GM-PHD filter, each target is updated by the measurement set $Z_{k}$. The updated weights of each target can be obtained as

$$
\begin{gathered}
w_{k}^{l, n}=p_{D, k} w_{k \mid k-1}^{l}\left(z_{k}^{n} ; \eta_{k \mid k-1}^{l}, S_{k}^{l}\right), l=1, \ldots, J_{k \mid k-1} \\
\bar{w}_{k, \text { updated }}^{l, n}=\frac{w_{k, \text { updated }}^{l, n}}{\kappa\left(z_{k}^{n}\right)+\sum_{i=1}^{J_{k \mid k-1}} w_{k, \text { updated }}^{i, n}} \\
\eta_{k \mid k-1}^{l}=H_{k} m_{k-1}^{l} \\
S_{k}^{l}=R_{k}+H_{k} P_{k \mid k-1}^{l} H_{k}^{T}
\end{gathered}
$$

where $\bar{w}_{k}^{l, n}$ and $w_{k}^{l, n}$ denote the normalized and original weight of target state $x_{k}^{l} \cdot H_{k}$ is the measurement matrix, and $R_{k}$ is the measurement noise covariance.

After update step of the GM-PHD filter, two matrices with the size $J_{k \mid k-1} \times N_{k}$ can be formed to store original weights and normalized weights of targets, which are called weight matrix $M_{w}$ and normalized weight matrix $\bar{M}_{w}$, respectively. In multi-target tracking, the targets and measurements should obey one-to-one rule as illustrated above. If the weight summation of Gaussian components with the same label is bigger than one in $\bar{M}_{w}$, it means that the rule is break by some targets, and the weights of the targets need to be reweighted.

The CPGM-PHD filter uses a collaborative penalized weight scheme to manage the weights of targets when targets move near each other. Unfortunately, the filter fails to deal with some targets that break one-to-one rule. The drawback of the CPGM-PHD filter is demonstrated by Fig.2. The normalized weight matrix $\bar{M}_{w}$ has three rows $l=1, l=2$ and $l=3$, where rows $l=2$ and $l=3$ belong to target 1 , and row $l=1$ belongs to target 2. In Fig.2(4), it can be seen that the weight $\bar{w}^{2,2}=0.9335$ is the maximum in current normalized weight matrix, and belongs to the target 1 . Therefore, the target 1 is firstly selected for reweighting. Due to the fact that the weight

$$
\begin{aligned}
& n=1 \quad n=2 \quad n=3 . \quad n=1 \quad n=2 \quad n=3
\end{aligned}
$$

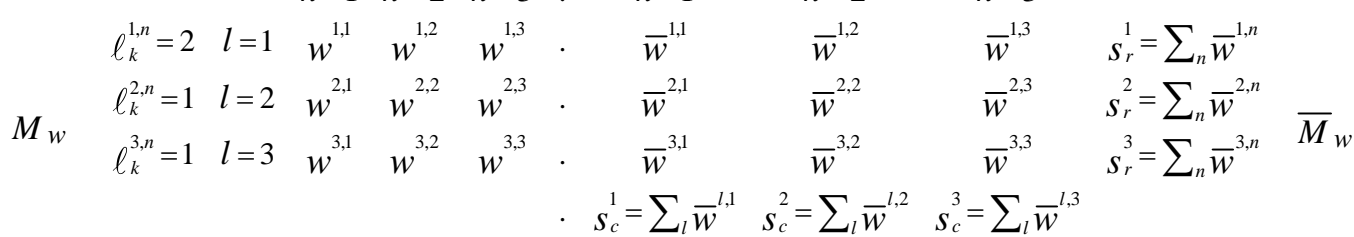

$$
\begin{array}{cccccccccc} 
& & n=1 & n=2 & n=3 & . & n=1 & n=2 & n=3 & \\
\ell_{k}^{1, n}=2 & l=1 & 0.0041 & 0.0239 & 0.1185 & . & 0.4184 & 0.0360 & 0.9215 & s_{r}^{1}=1.3759 \\
\ell_{k}^{2, n}=1 & l=2 & 0.0002 & 0.6721 & 0.0017 & . & 0.0204 & 0.9335 & 0.0132 & s_{r}^{2}=0.9671 \\
\ell_{k}^{3, n}=1 & l=3 & 0.0055 & 0.0220 & 0.0084 & . & 0.5612 & 0.0305 & 0.0653 & s_{r}^{3}=0.657
\end{array}
$$

(4)

$\begin{array}{cccccccccc} & & n=1 & n=2 & n=3 & . & n=1 & n=2 & n=3 & \\ \ell_{k}^{1, n}=2 & l=1 & 0.0003 & 0.0019 & 0.1185 & . & 0.05 & 0.0027 & 0.9215 & s_{r}^{1}=0.9742 \\ \ell_{k}^{2, n}=1 & l=2 & 0.0002 & 0.6721 & 0.0017 & . & 0.0333 & 0.9657 & 0.0132 & s_{r}^{2}=1.0122 \\ \ell_{k}^{3, n}=1 & l=3 & 0.0055 & 0.0220 & 0.0084 & . & 0.9167 & 0.0316 & 0.0653 & s_{r}^{3}=1.0136 \\ & & & & & . & s_{c}^{1}=1 & s_{c}^{2}=1 & s_{c}^{3}=1 & \end{array}$

Fig. 2. The process of weights punishment in the CPGM-PHD filter 
summation of Gaussian components of the row $l=2$ in $\bar{M}_{w}$ is $s_{r}^{2}=0.9671$, the target 1 is considered to be correct in the CPGM-PHD filter, and will not be reweighted. Then, the target 2 is selected for next reweighting, and the reweighted result of Gaussian components of the row $l=1$ is shown in Fig.2(6). Owning to fact that there is no other targets in current normalized weight matrix, the process of weights punishment in the CPGM-PHD filter terminates. However, the rows $l=2$ and $l=3$ belong to target 1 , the weight summation of the target is 1.6241 in Fig.2(4), which is bigger than one. According to one-to-one rule in multi-target tracking, the weights of the target need to be reweighted. Unfortunately, the collaborative penalized weight scheme of the CPGM-PHD filter does not reweights the weights of the target 1 .

To solve the drawback of the CPGM-PHD filter and improve the estimation accuracy of closely spaced targets, a novel reweighting scheme is incorporated into the framework of the GM-PHD filter.

At the end of each update step of the GM-PHD filter, the novel reweighting scheme starts to work. A set $\Xi_{S L}$ is initialized with null, and the details of the novel reweighting scheme are described as follows.

(1) Gaussian component with the maximum weight in current normalized weight matrix is obtained as

$$
\left[l^{*}, n^{*}\right]=\underset{l \in I \text { and } \forall n=1:_{N k}}{\arg \max }\left(\bar{w}_{k}^{l, n}\right), I=\left\{1, \ldots, J_{k \mid k-1}\right\}-\Xi_{S L}
$$

where $J_{k \mid k-1}$ is the number of Gaussian components.

(2) Gaussian components, having the same label as Gaussian component with maximum weight, are selected, and weight summation of these components can be obtained as

$$
\begin{aligned}
& \Xi_{S L}=\left\{i \mid \ell_{k-1}^{i}=\ell_{k-1}^{l^{*}}, i \in I\right\} \\
& \delta=\sum_{n=1}^{N_{k}} \bar{w}_{k}^{l^{*} \cdot n}, \forall l^{*} \in \Xi_{S L}
\end{aligned}
$$

(3) If $\delta>1$, the weights of the target should be redistributed as

$$
\begin{aligned}
& \beta=\lambda \times(1-\delta / \mu) \times\left(1-\bar{w}_{k}^{l^{*}, n^{n}}\right)
\end{aligned}
$$

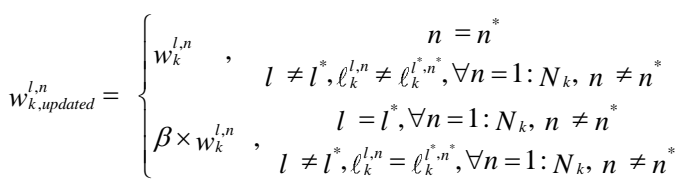

$$
\begin{aligned}
& \bar{w}_{k, \text { tupdated }}^{l, n}=\frac{w_{k, u p p d a t e d}^{l, n}}{\kappa_{k}\left(z_{k}^{n}\right)+\sum_{i=1}^{J_{\text {klk-1 }}} w_{k, \text { updated }}^{i, n}}
\end{aligned}
$$

where $\mu$ is the number of Gaussian components of the target, and scaling factor $\lambda$ is distributed uniformly on the interval $[0,1]$. Then, go to (2). Otherwise, the target needs not to be reweighted.

(4) If $I \neq N U L L$, then go to (1). Otherwise, the novel reweighting scheme terminates.

The main steps of the proposed algorithm implementation are summary as follows.

Prediction: Suppose that, at time $k-1$, the posterior multi-target intensity is given, which can be approximated by Eq.(3). At time $k$, the newborn target intensity can be obtained as

$$
\gamma_{k}(x)=\sum_{i=1}^{J_{\gamma, k}} w_{\gamma, k}^{(i)} \quad\left(x ; m_{\gamma, k}^{(i)} ; p_{\gamma, k}^{(i)}\right)
$$

Then the predicted multi-target intensity $v_{k \mid k-1}$ can be obtained as

$$
\begin{gathered}
\mathcal{V}_{k \mid k-1}=\sum_{i=1}^{J_{k k-1}} w_{k \mid k-1}^{(i)}\left(x ; m_{S, k \mid k-1,}^{(i)} P_{S, k \mid k-1,}^{(i)}\right)+\gamma_{k}(x) \\
w_{k \mid k-1}^{(i)}=p_{S, k} w_{k-1}^{(i)} \\
m_{S, k \mid k-1}^{(i)}=F_{k-1} m_{S, k-1}^{(i)} \\
P_{S, k \mid k-1}^{(i)}=F_{k-1} P_{S, k-1}^{(i)} F_{k-1}^{T}+Q_{k-1}
\end{gathered}
$$

where $F_{k-1}$ is the state transition matrix, and $Q_{k-1}$ is the process noise covariance. Each newborn target is given a new label. A label set is form by concatenating the prediction labels and newborn target labels, which can be obtain as

$$
L_{k \mid k-1}=L_{k-1} \cup\left\{\ell_{\gamma, k}^{1}, \ldots, \ell_{\gamma, k}^{i}, \ell_{\gamma, k}^{J_{\gamma, k}}\right\}, i \in\left[1, J_{\gamma, k}\right]
$$

Update : when the measurement set $Z_{k}=\left\{z_{n, k}\right\}_{n=1}^{N_{k}}$ is available at time $k$, the multi-target posterior intensity $v_{k}$ can be obtained as Eq.(5), the updated weight $w_{k}^{(i)}(z)$ is computed by Eq.(6), where the likelihood function $g(\square)$, the updated mean $m_{k \mid k}^{(i)}$ and updated covariance $P_{k \mid k}^{(i)}$ can be approximated as

$$
\begin{gathered}
g\left(z \mid x^{(i)}\right)=\left(z ; \hat{z}_{k \mid k-1}^{(i)}, R_{k}+H_{k} P_{k \mid k-1}^{(i)} H_{k}^{T}\right) \\
m_{k \mid k}^{(i)}(z)=m_{k \mid k-1}^{(i)}+K_{k}^{(i)}\left(z-\hat{z}_{k \mid k-1}^{(i)}\right) \\
\hat{z}_{k \mid k-1}^{(i)}=H_{k} m_{k \mid k-1}^{(i)} \\
P_{k \mid k}^{(i)}=\left[I-K_{k}^{(i)} H_{k}\right] P_{k \mid k-1}^{(i)} \\
K_{k}^{(i)}=P_{k \mid k-1}^{(i)} H_{k}^{T}\left(R_{k}+H_{k} P_{k \mid k-1}^{(i)} H_{k}^{T}\right)^{-1}
\end{gathered}
$$

There are $\left(1+N_{k}\right) J_{k \mid k-1}$ Gaussian components generated in the update step of the GM-PHD filter, where each prediction item has $1+N_{k}$ Gaussian components. The updated label set can be obtained by assigning the same label as the related predicted components

$$
L_{k}=L_{k}^{V_{k \mid k-1}} \cup L_{k}^{z 1} \cup \ldots \cup L_{k}^{z_{N k}}
$$

Then, the reweighting scheme starts to work as demonstrated above.

Fig.3 illustrates one of the reweighting processes of the proposed algorithm. In Fig.3(2), $\bar{w}^{2,2}=0.9335$ is the maximum in current normalized weight matrix, meanwhile rows $l=2$ and $l=3$ belong to target 1 , and the weight summation of target 1 is $s_{r}^{2}+s_{r}^{3}=1.6241$. Therefore, the weights of Target 1 should be reweighted. The weights of the two rows in original weight matrix are firstly rectified by the novel reweighting scheme, and then renormalized the original weights matrix. The updated original weight matrix and updated normalized weight matrix are shown in Fig.3(3) and Fig.3(4), respectively. In Fig.3(4), the weight summation of target 1 is $s_{r}^{2}+s_{r}^{3}=0.9893$, so the reweighting process of target 1 stops and the novel reweighting scheme continues to dispose the remaining targets. As seen, the weight summation of target 


$\begin{array}{cccccccccc} & & n=1 & n=2 & n=3 & . & n=1 & n=2 & n=3 & \\ \ell_{k}^{1, n}=2 & l=1 & 0.0041 & 0.0239 & 0.1185 & . & 0.4184 & 0.0360 & 0.9215 & s_{r}^{1}=1.3759 \\ \ell_{k}^{2, n}=1 & l=2 & 0.0002 & 0.6721 & 0.0017 & . & 0.0204 & 0.9335 & 0.0132 & s_{r}^{2}=0.9671 \\ \ell_{k}^{3, n}=1 & l=3 & 0.0055 & 0.0220 & 0.0084 & . & 0.5612 & 0.0305 & 0.0653 & s_{r}^{3}=0.657 \\ & & & & & . & s_{c}^{1}=1 & s_{c}^{2}=1 & s_{c}^{3}=1 & \end{array}$

(1)

$$
\begin{array}{cccc} 
& & n=1 & n=2 \\
\ell_{k}^{1, n}=2 & l=1 & 0.0041 & 0.0239 \\
\ell_{k}^{2, n}=1 & l=2 & 0.0 & 0.6721 \\
\ell_{k}^{3, n}=1 & l=3 & 0.0001 & 0.0220
\end{array}
$$$$
n=3
$$$$
0.1185
$$$$
n=1
$$

0.00003

0.9762

$n=2$

$$
0.0
$$

0.0360

$n=3$

$$
\begin{array}{cccc} 
& & n=1 & n=2 \\
\ell_{k}^{1, n}=2 & l=1 & 0.000004 & 0.00002 \\
\ell_{k}^{2, n}=1 & l=2 & 0.0 & 0.6721 \\
\ell_{k}^{3, n}=1 & l=3 & 0.0001 & 0.0220
\end{array}
$$$$
n=3
$$$$
0.0238
$$

0.9335

0.0305

0.9985

0.0002

$$
s_{c}^{1}=1
$$$$
s_{c}^{2}=1
$$

0.0013

$s_{r}^{1}=2.0107$

(4)$$
0.1185
$$$$
n=1
$$$$
n=2
$$$$
s_{c}^{3}=1
$$

$s_{r}^{2}=0.9337$

$s_{r}^{3}=0.0556$

0.00003

\section{$\begin{array}{lll}0.0385 & 0.00002 & 0.9985\end{array}$}

$\begin{array}{lll}0.0 & 0.9683 & 0.0002\end{array}$

$s_{r}^{1}=1.03702$$$
\begin{array}{lll}
0.9615 & 0.03168
\end{array}
$$

$$
S_{c}^{1}=1 \quad S_{c}^{2}=1 \quad S_{c}^{3}=1
$$

$$
\begin{array}{cccccccccc} 
& & n=1 & n=2 & n=3 & . & n=1 & n=2 & n=3 & \\
\ell_{k}^{1, n}=2 & l=1 & 0.0 & 0.0 & 0.1185 & . & 0.0 & 0.0 & 0.9985 & s_{r}^{1}=0.9985 \\
\ell_{k}^{2, n}=1 & l=2 & 0.0 & 0.6721 & 0.00003 & . & 0.0 & 0.9683 & 0.0002 & s_{r}^{2}=0.9685 \\
\ell_{k}^{3, n}=1 & l=3 & 0.0001 & 0.0220 & 0.00015 & . & 1 & 0.0317 & 0.0013 & s_{r}^{3}=1.033 \\
& & & & & . & s_{c}^{1}=1 & s_{c}^{2}=1 & s_{c}^{3}=1 &
\end{array}
$$

Fig. 3. The reweighting process of the proposed algorithm

new original weight matrix and normalized weight matrix are shown in Fig.3(5) and 3(6). However, the weight summation of target 2 is $s_{r}^{1}=1.03702$ in Fig.3(6), which is not meet the one-to-one rule, and needs to be reweighted again. After second reweighting target 2 , the original weight matrix and normalized weight matrix are shown in Fig.3(7) and 3(8). In Fig.3(8), the weight summation of target 2 is $s_{r}^{1}=0.9985$, which has reached a reasonable scope. By combining the rows $l=2$ and $l=3$ in Fig.3(4) and the row $l=1$ in Fig.3(8), a new normalized weight matrix can be formed. Because each target can obey the one-to-one rule in the new normalized weight matrix, the process of the novel reweighting scheme dealing with closely spaced targets terminates at current time step.

\section{Simulation Results}

In this section, the effectiveness of the proposed algorithm is studied by multi-target tracking experiment compared to the existing relevant methods. Suppose, targets move in a two dimensional plane with $[-500,500] \times[-500,500]\left(\mathrm{m}^{2}\right)$. At time $k$, state vector of each target $X_{k}=\left[x_{k}, y_{k}, v_{x_{k}}, v_{y_{k}}\right]^{T}$ is composed of position $\left[x_{k}, y_{k}\right]^{T}$ and velocity $\left[v_{x_{k}}, v_{y_{k}}\right]^{T} . T=1 \mathrm{~s}$ is the sample interval, and all simulation scenarios are 100 times. Each target follows a linear Gaussian dynamical model and sensor has a linear Gaussian measurement model, i.e.,

$$
\begin{gathered}
f_{k \mid k-1}(x \mid \xi)=\left(x ; F_{k-1} \xi, Q_{k-1}\right) \\
g_{k \mid k-1}(z \mid x)=\left(x ; H_{k} x, R_{k}\right)
\end{gathered}
$$

where the process noise covariance matrix $Q=\operatorname{diag}([0.5,0.5])$ and the measurement noise matrix $R=\operatorname{diag}([2500,2500])$, respectively.

The survival probability $p_{S, k}=0.99$ and detection probability $p_{D, k}=0.99$. The intensity of new birth targets RFS is given by

$$
\gamma_{k}(x)=\sum_{i=1}^{3} 0.1 \quad\left(x ; m_{\gamma}^{(i)}, P_{\gamma}\right)
$$

where $P_{\gamma}=\operatorname{diag}([100,100,25,25])$, and $m_{\gamma}^{(i)}$ is different according to different scenario. 
The pruning threshold $T_{t h}=0.00001$, the merging threshold $U=4$, and the maximum number of Gaussian components $T_{\max }=100$.

To effectively evaluate the performance of different algorithms, 200 Monte Carlo runs are performed. Three criteria are adopted, which are the estimated target number, the mean number of targets estimation error (NTE) [14], and the optimal sub-pattern assignment (OSPA) [15]. The OSPA distance and NTE can be described as

$$
\begin{gathered}
\operatorname{NTE}\left\{X_{k}, \hat{X}_{k}\right\}=E\left\{\left|\hat{X}_{k}\right|-\left|X_{k}\right|\right\} \\
O S P A_{p, c}\left(X_{k}, \hat{X}_{k}\right)=\frac{1}{\left|\hat{X}_{k}\right|} 1 / p \\
\left(\min _{\left.\pi \in \prod_{\left|\hat{X}_{k}\right|} \sum_{i=1}^{\left|X_{k}\right|}\left(d_{c}\left(x^{i}, \hat{x}^{\pi(i)}\right)\right)^{p}+c^{p} \times\left(\left|\hat{X}_{k}\right|-\left|X_{k}\right|\right)\right)^{1 / p}}^{1 / 3}\right.
\end{gathered}
$$

where parameters $X_{k}$ and $\hat{X}_{k}$ denote the true set and estimated set of target. The parameters $p$ and $c$ are set to 2 and 100 , respectively.

Scenario 1. Multi-target with crossing motion

Fig.4 shows a simulated scenario with three crossing targets, where the clutter rate is modeled as a Poisson RFS with the mean $\lambda_{c}=5 \times 10^{6} \mathrm{~m}^{-2}$.

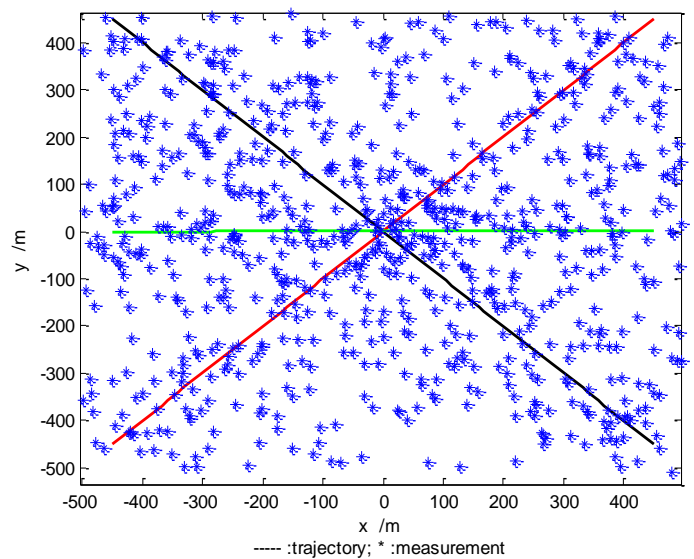

Fig. 4. Three targets with crossing motion scenario

Fig.5 illustrates the comparison result of different algorithms in crossing target scenario. As seen, the PGM-PHD filter, the CPGM-PHD filter and proposed algorithm outperform the GM-PHD filter in OSPA distance, NTE and estimated target number. Owning to the fact that the proposed algorithm is able to effectively solve the problem of closely spaced targets, it achieves more excellent performance. The lower OSPA distance means that the proposed algorithm has higher estimation accuracy. However, there is a peak of the OSPA distance between time 50 and 70 from the proposed algorithm shown in Fig.5(a). The reason is that the estimation accuracy of the proposed algorithm is relatively low when targets move near each other.

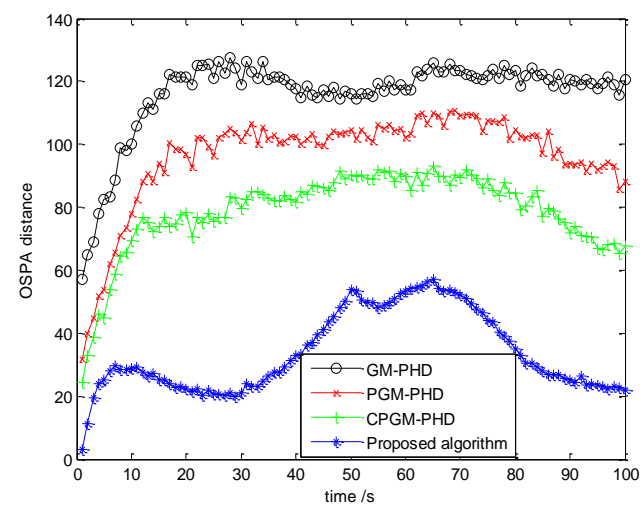

(a) OSPA distance

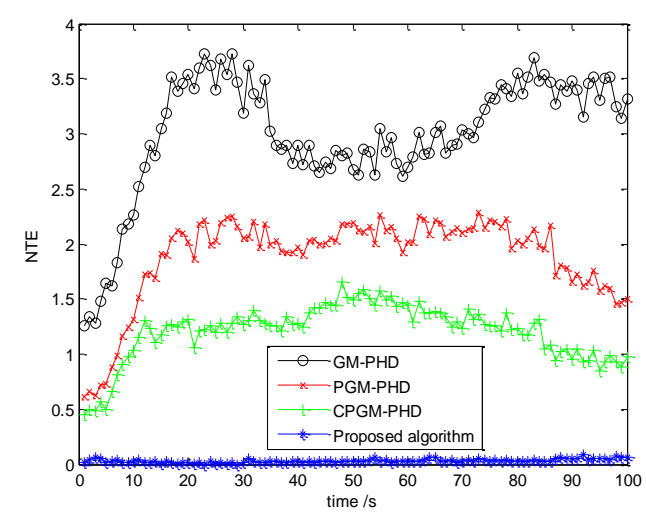

(b) NTE

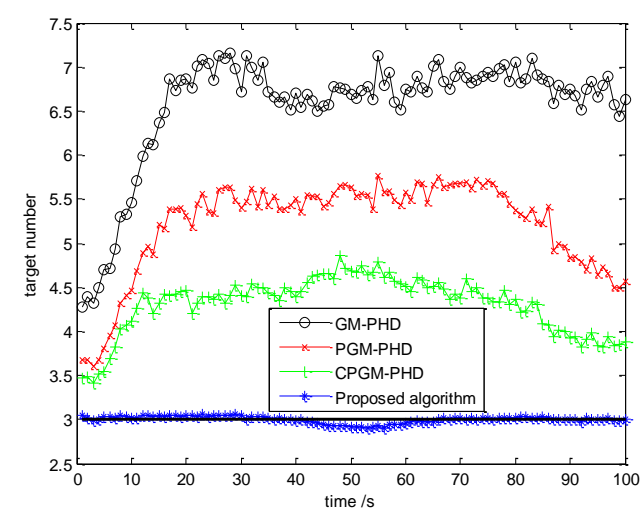

(c) Estimated target number

Fig. 5. Simulation results of three crossing targets

Scenario 2. Multi-target with paralleling motion

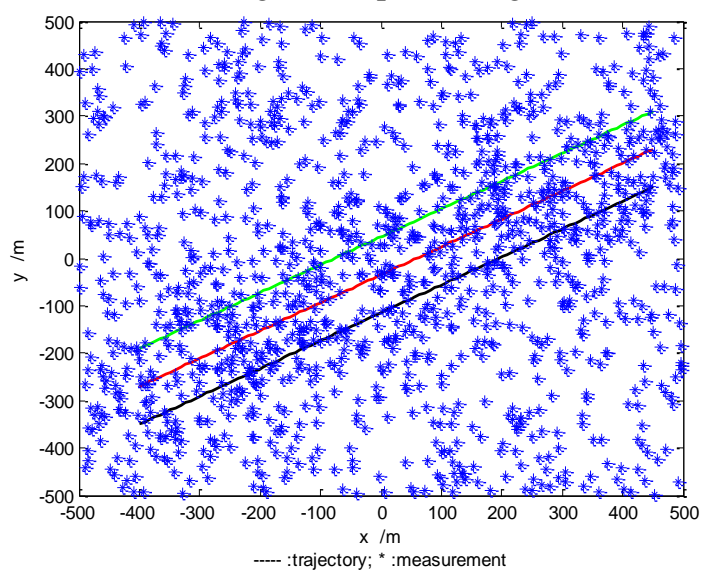

Fig. 6. Three targets with paralleling motion scenario 


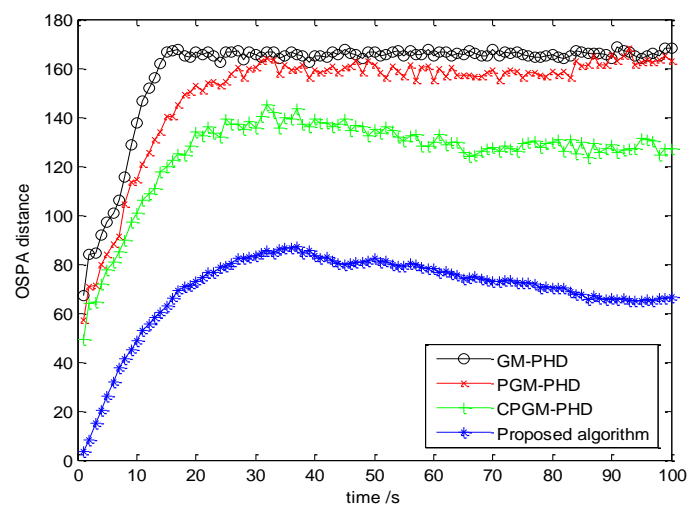

(a) OSPA distance

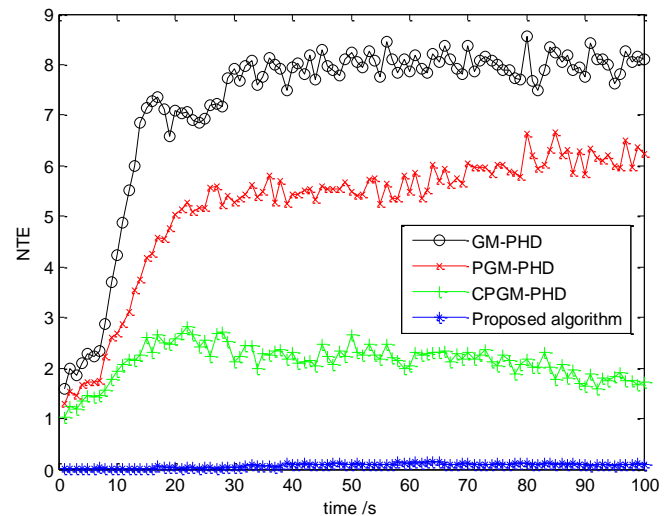

(b) NTE

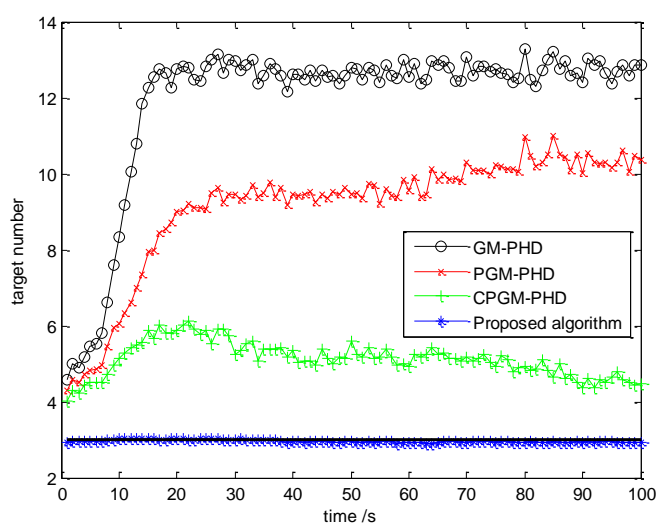

(c) Estimated target number

Fig. 7. Simulation results of three paralleling targets

As is shown in Fig.6, three paralleling targets are simulated, and the clutter rate is modeled as a Poisson RFS with the mean $\lambda_{c}=10 \times 10^{6} \mathrm{~m}^{-2}$.

Scenario 3. Multi-target cross or parallel under different clutter rates

In order to study the effective of the proposed algorithm in unknown and complex tracking environment, two experiments are carried out from different clutter rates to compare the performance of different algorithms. One of the experiments adopts multiple targets with crossing motion scenario, and another adopts multiple targets with paralleling motion scenario. The OPSA distance and NTE are utilized to evaluate the performance of different algorithms.

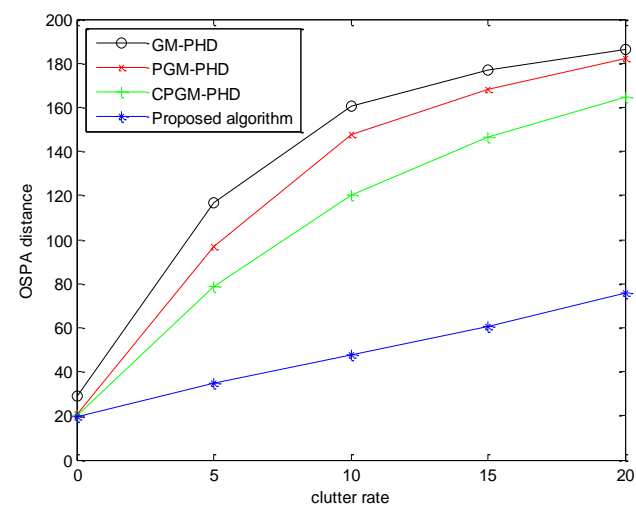

(a) OSPA distance

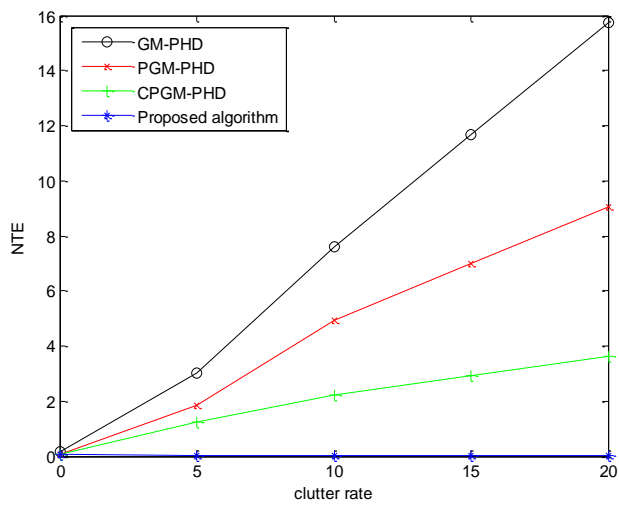

(b) NTE

Fig. 8. Simulation results of different clutter rates in three crossing target scenario

The clutter rate $\lambda_{c}$ varies from 0 to 20 with the interval of 5 in each clutter rate test. The detection probability $p_{D, k}=0.98$, and the other parameters are the same as those of scenarios 1 and 2 , respectively.

Fig.8 shows the OSPA and NTE criteria of the four algorithms in three crossing targets scenario with different clutter rates. As the clutter rate increased, the disturbance of the clutters is more seriously, which makes both the OSPA distance and NTE obtained from the four algorithms have been improved to some extent. However, the proposed algorithm illustrates its excellent tracking performance, where the OSPA distance and NTE increase little far less than that of the other filters. It can be concluded that the proposed algorithm has a better performance in both the target number estimation and their states, which is favourable for tracking closely spaced targets.

The comparison results of OSPA distance and NTE of different algorithms are shown in Fig.9, in which three paralleling targets are simulated in a scenario with varied clutter rates. It can be seen that the performance of the proposed algorithm outperform the GM-PHD, PGM-PHD and CPGM-PHD filters again in paralleling target scenario with various clutter rates. Due to the novel reweighting scheme, both the OSPA distance and NTE of proposed algorithm have great advantages. Specifically, the OSPA distance of the proposed algorithm increases slowly as significant increase in clutter rate shown in Fig.9(a), Meanwhile the NTE of the proposed 
algorithm remains almost zero even though clutter rate increased significantly. The better performance of the proposed algorithm means it has strong robust without disturbed by clutter in complex tracking environment.

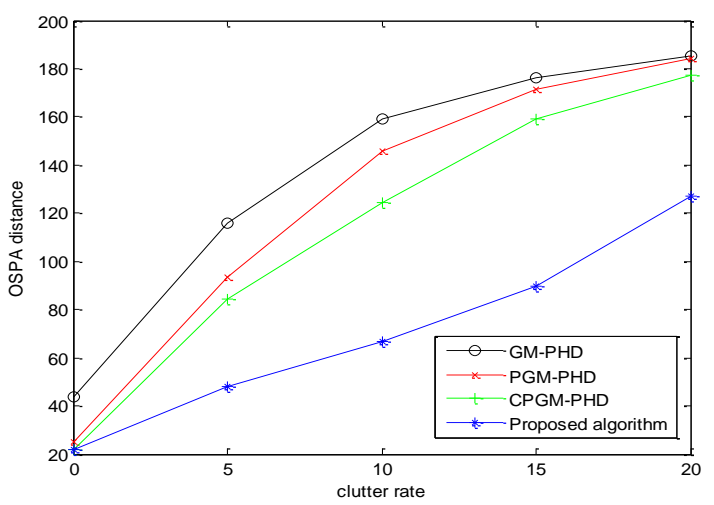

(a) OSPA distance

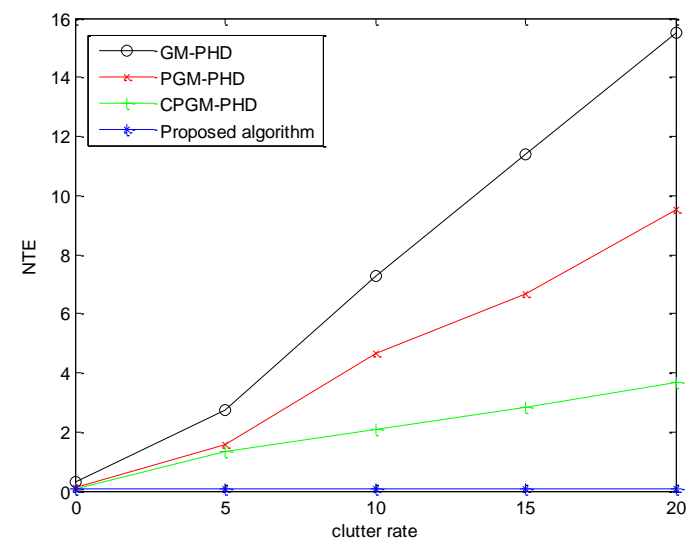

(a) NTE

Fig.9. Simulation results of different clutter rates in three paralleling target scenario

\section{CONCLUSIONS}

For tracking closely spaced targets, a novel reweighting scheme is introduced under the framework of the GM-PHD filter. Based on the reweighting scheme, the weight error of closely spaced targets can be properly alleviated. The numerical studies show that the proposed algorithm can accurately estimate the number of target and their states in unknown and complex tracking environment.

\section{REFERENCES}

[1] R. Mahler, Statistical multisource multitarget information fusion, Artech House, Norwood, MA, 2007.

[2] R. Mahler, Multitarget Bayes filtering via first-order multitarget moments, IEEE Transaction on Aerospace and Electronic Systems, Vol.39, No.4, 2003, pp.1152-1178.

[3] R. Mahler, PHD filters of higher order in target number, IEEE Transactions on Aerospace and Electronic Systems, Vol.43, No.4, 2007, pp.1523-1543.

[4] B.N. Vo, S. Singh, A. Doucet, Sequential Monte Carlo implementation of the PHD filter for multi-target tracking, Proc. of the 6th International Conference on Information Fusion, 2003, pp.792-799.

[5] B.N. Vo, W.K. Ma, The Gaussian mixture probability hypothesis density filter, IEEE Transactions on Signal Processing, Vol.54, No.11, 2006, pp.4091-4104.

[6] C. OuYang, H.B. Ji, Y. Tian, Improved Gaussian mixture CPHD tracker for multitarget tracking, IEEE Transaction on Aerospace and Electronic Systems, Vol.49, No.2, 2013, pp.1177-1191.

[7] T.C. Li, S.D. Sun, T.P. Sattar, High-speed Sigma-gating SMC-PHD filter, Signal Processing, Vol.93, No.9, 2013, pp.2586-2593.

[8] B. Li, F.W. Pang, Improved cardinalized probability hypothesis density filtering algorithm, Applied Soft Computing, Vol.24, 2014, pp.692-703.

[9] M. Yazdian-Dehkordi, O.R. Rojhani, Z. Azimifar, Visual target tracking in occlusion condition: A GM-PHD-based approach, Proc. of 16th CSI International Symposium on Artificial Intelligence and Signal Processing (AISP), 2012, pp. 538-541.

[10] S. Reuter, B.T. Vo, B.N. Vo, et al., The Labeled Multi-Bernoulli Filter, IEEE Transactions on Signal Processing, Vol.62, No.12, 2014, pp:3246-3260.

[11] B.T. Vo, B.N. Vo, Labeled Random Finite Sets and Multi-Object Conjugate Priors, IEEE Transactions on Signal Processing, Vol.61, No.13, 2013, pp.3460-3475.

[12] B.N. Vo, B.T. Vo, D. Phung, Labeled Random Finite Sets and the Bayes Multi-Target Tracking Filter, IEEE Transactions on Signal Processing, Vol.62, No.24, 2014, pp.6554-6567.

[13] M. Yazdian-Dehkordi, Z. Azimifar, M.A. Masnadi-Shirazi, An improvement on GM-PHD filter for occluded target tracking, Proc. of the IEEE 36th International Conference on Acoustics, Speech and Signal Processing (ICASSP), 2011, pp.1773-1776.

[14] M. Yazdian-Dehkordi, Z.Azimifar, M.A. Masnadi-Shirazi, Penalized Gaussian mixture probability hypothesis density filter for multiple target tracking, Signal Processing, Vol.92, No.5, 2012, pp.1230-1242.

[15] Y. Wang, H.D. Meng, Y.M Liu, et al., Collaborative penalized Gaussian mixture PHD tracker for close target tracking, Signal Processing, Vol.102, 2014, pp:1-15.

[16] M. Yazdian-Dehkordi, Z. Azimifar, M.A. Masnadi-Shirazi, Competitive Gaussian mixture probability hypothesis density filter for multiple target tracking in the presence of ambiguity and occlusion, IET Radar, Sonar \& Navigation, Vol.6, No.4, 2012, pp.251-262.

[17] D. Schuhmacher, B.T. Vo, B.N. Vo, A Consistent Metric for Performance Evaluation of Multi-Object Filters, IEEE Transactions on Signal Processing, Vol.56, No.8, 2008, pp.3447-3457. 